\title{
Pathologic tearfulness after limbic encephalitis
}

\section{A novel disorder and its neural basis}

\author{
Georgios P.D. Argyropoulos, PhD, Lauren Moore, BSc, Clare Loane, PhD, Adriana Roca-Fernandez, MSc, \\ Carmen Lage-Martinez, MD, Oana Gurau, MSc, Sarosh R. Irani, FRCP, PhD, Adam Zeman, FRCP, and \\ Christopher R. Butler, FRCP, PhD
}

Neurology ${ }^{\circledR}$ 2020;94:e1320-e1335. doi:10.1212/WNL.0000000000008934

\section{Abstract}

\section{Objective}

We investigated the nature and neural foundations of pathologic tearfulness in a uniquely large cohort of patients who had presented with autoimmune limbic encephalitis (aLE).

\section{Methods}

We recruited 38 patients ( 26 men, 12 women; median age 63.06 years; interquartile range [IQR] 16.06 years) in the postacute phase of aLE who completed questionnaires probing emotion regulation. All patients underwent structural/functional MRI postacutely, along with 67 age- and sex-matched healthy controls (40 men, 27 women; median age 64.70 years; IQR 19.87 years). We investigated correlations of questionnaire scores with demographic, clinical, neuropsychological, and brain imaging data across patients. We also compared patients diagnosed with pathologic tearfulness and those without, along with healthy controls, on gray matter volume, resting-state functional connectivity, and activity.

\section{Results}

Pathologic tearfulness was reported by $50 \%$ of the patients, while no patient reported pathologic laughing. It was not associated with depression, impulsiveness, memory impairment, executive dysfunction in the postacute phase, or amygdalar abnormalities in the acute phase. It correlated with changes in specific emotional brain networks: volume reduction in the right anterior hippocampus, left fusiform gyrus, and cerebellum, abnormal hippocampal resting-state functional connectivity with the posteromedial cortex and right middle frontal gyrus, and abnormal hemodynamic activity in the left fusiform gyrus, right inferior parietal lobule, and ventral pons.

\section{Conclusions}

Pathologic tearfulness is common following aLE, is not a manifestation of other neuropsychiatric features, and reflects abnormalities in networks of emotion regulation beyond the acute hippocampal focus. The condition, which may also be present in other neurologic disorders, provides novel insights into the neural basis of affective control and its dysfunction in disease.

\author{
Correspondence \\ Dr. Argyropoulos \\ georgios.argyropoulos@ \\ ndcn.ox.ac.uk
}

\section{MORE ONLINE}

ค Podcast

Dr. Stacey Clardy talks with

Dr. Chris Butler about his

paper on pathologic tearfulness after limbic encephalitis.

NPub.org/965c4u

\section{- CME Course}

NPub.org/cmelist 


\section{Glossary}

aLE $=$ autoimmune limbic encephalitis; ANOVA $=$ analysis of variance; $\mathbf{B A}=$ Brodmann area $\mathbf{B I S}=$ Barratt Impulsiveness Scale; $\mathbf{B O L D}=$ blood oxygenation level-dependent; CBS = Cambridge Behaviour Scale; CNS-LS = Center for Neurologic Study-Lability Scale; DARTEL = diffeomorphic anatomical registration through the exponentiated lie algebra; EPI = echoplanar imaging; FWE = family-wise error; FWHM = full width at half maximum; GM = gray matter; HADS = Hospital Anxiety and Depression Scale; HC = healthy control; IQR = interquartile range; IRQ = Irritability Questionnaire; MAP = Memory and Amnesia Project; MNI = Montreal Neurologic Institute; MTL = medial temporal lobe; MVPA = multivariate pattern analysis; OPTIMA = Oxford Project to Investigate Memory and Ageing; PCA = principal component analysis; rsALFF = resting-state abnormalities in the local amplitude of low-frequency fluctuations; rsFC = resting-state functional connectivity; rsfMRI = resting-state fMRI; TIV = total intracranial volume; VBM = voxel-based morphometry; $\mathbf{W M}=$ white matter.

Most neurologic research on emotion dysregulation focuses on pseudobulbar affect, which occurs in a broad range of disorders with diffuse or poorly characterized pathology ${ }^{1-5}$ often implicating the brainstem and cerebellum. ${ }^{1,6-8}$ The fact that pseudobulbar affect has not been associated with focal limbic damage is consistent with its being understood as "a disorder of emotional expression rather than a primary disturbance of feelings." 7

Autoimmune limbic encephalitis (aLE) is associated with the subacute onset of amnesia and seizures and high T2signal (acute MRI) in the limbic system, especially the hippocampus. Patients often respond satisfactorily to immunosuppressive therapy, ${ }^{9}$ although many develop hippocampal atrophy and residual cognitive impairment. ${ }^{10,11}$ While behavioral/psychiatric symptoms may occur acutely, ${ }^{12}$ persisting problems with readily provoked tearfulness are only mentioned in passing, ${ }^{13-15}$ and we have encountered complaints of such symptoms among many of our patients.

We aimed to determine the so far unexplored nature and neural correlates of pathologic tearfulness following aLE in a uniquely large cohort of patients $(\mathrm{n}=38)$. We investigated its relationships with demographic and clinical data, selfreported measures of emotion regulation, and performance on neuropsychological tests. We hypothesized that it is associated with abnormalities in the hippocampus, the amygdala, hippocampal-diencephalic-cingulate networks, and cerebro-ponto-cerebellar loops: aLE results in relatively focal hippocampal atrophy, ${ }^{11,16}$ and the limbic system is involved in emotion processing. ${ }^{17-19}$ Amygdala abnormalities are sometimes observed ${ }^{20}$ and have been associated with abnormal autonomic arousal. ${ }^{21}$ Furthermore, the hippocampus is embedded within broader hippocampaldiencephalic-cingulate networks supporting emotion regulation. ${ }^{22}$ We have recently shown abnormalities in this extended circuitry in aLE. ${ }^{23,24}$ Finally, in a prominent pathophysiologic account, emotion dysregulation in pseudobulbar affect was caused by disruption to cerebro-pontocerebellar pathways, ${ }^{7}$ with which the hippocampus communicates. $^{25}$

\section{Methods}

\section{Standard protocol approvals, registrations, and patient consents}

Ethical approval was received from the South Central Oxford Research Ethics Committee (REC no. 08/H0606/133). All participants provided written informed consent according to the Declaration of Helsinki.

\section{Participants}

We report data relating to pathologic tearfulness in 38 patients with aLE ( 26 male, 12 female; median age at research MRI 63.06 years; interquartile range [IQR] 16.16 years $)^{24}$ after the acute stage of the disease (median 5.41; IQR 5.36 years since symptom onset). All patients were fluent in English (37 native speakers; 1 non-native speaker) and had undergone MRI at the time of initial clinical presentation as well as neuropsychological assessment at the Russell Cairns Unit, Oxford, UK (2013-2018).

All patients had been diagnosed with aLE according to established diagnostic criteria ${ }^{26}$ : (a) subacute symptom onset suggesting involvement of the limbic system; (b) bilateral abnormalities restricted within the medial temporal lobes (MTLs) on T2-weighted MRI; (c) CSF pleocytosis (white blood cells $>5 / \mathrm{mm}^{3}$ ) or slow-wave/epileptic activity involving the temporal cortex (EEG); (d) exclusion of alternative causes (e.g., CNS infections/drug toxicity/stroke/ Creutzfeldt-Jakob disease/Kleine-Levin syndrome, mitochondrial/neoplastic/epileptic/rheumatologic disorders, septic/metabolic encephalopathy); (e) antibodies against cell-surface/synaptic/onconeural proteins. Criteria $(a-d)$ are required for a diagnosis of definite limbic encephalitis, unless, in the absence of one of $(\mathrm{a}-\mathrm{c})$, criterion (e) is satisfied. ${ }^{26}$

A total of 34 of 38 patients satisfied the criteria for a diagnosis of definite aLE; the remaining $4 / 38$ had been diagnosed with aLE, meeting criteria (a, b, d), but not (e). No data could be recovered regarding (c). In 28/38 patients, an aLE-associated autoantibody was identified. A total of $10 / 38$ patients demonstrated the clinical profile of aLE with no identified antibody; such cases are well-recognized ${ }^{27}$ and are generally 
thought to involve antibodies not detected in clinical practice at the time of screening. No patient presented with positive PCR testing for herpes simplex virus or with anti-NMDAR encephalitis. ${ }^{28}$ Two of 38 patients had neoplastic lesions, thought to be the triggers for their autoimmune disorder, which were treated and were in full remission at the time of study participation. A total of 31/38 patients had been treated acutely with immunotherapy (e.g., plasma exchange, IV or oral prednisolone). A total of $34 / 38$ patients had shown abnormal hippocampal signal, volume, or diffusion on clinical MRI conducted acutely. Six of 38 patients showed amygdala abnormalities, 1 in the parahippocampal cortex, 1 in the entorhinal cortex, 4 patients had mild microangiopathic changes in keeping with their age, and 1 patient showed extraMTL abnormalities (bright caudate). No acute abnormalities were detected in $4 / 38$ patients, who nonetheless demonstrated clinical features characteristic of aLE; 35/38 patients had presented acutely with seizures.

Moreover, patients had no history of previous neurologic or psychiatric disorder that could have resulted in cognitive impairment. They were assessed by a single neurologist (CRB) prior to study inclusion. Their (acute) clinical and (postacute) neuropsychological details have been presented previously. ${ }^{24}$ Healthy controls (HCs) were recruited through the Oxford Project to Investigate Memory and Ageing (OPTIMA) and through local advertisement.

\section{Neuropsychological profile}

Postacutely, all patients and $57 \mathrm{HCs}$ ( 38 men, 19 women; age at assessment: median, 61.50; IQR 17.26 years; HCs vs patients: male:female ratio: $\chi^{2}=0.032, p=0.858$; age at assessment: $U=933.50, p=0.258$ ) underwent neuropsychological assessment. Patients showed preserved executive function, above-average premorbid intelligence, and spared motor, executive, and visuospatial function, but impaired episodic memory. ${ }^{24}$

\section{Review of medical records}

Details were extracted from medical records and interviews with the patients and caregivers using a standard proforma regarding clinical history, acute aLE presentation, and subsequent clinical course of each patient (age at symptom onset; presenting symptoms; premorbid and acute phase depression, anxiety, agitation, obsessionality, or hallucinations; seizure occurrence/recency; delay between symptom onset and start of treatment; autoantibody type; past/present immunotherapy, antiepileptics, and antidepressants).

\section{Emotion regulation assessment}

\section{Questionnaires}

In order to assess patients' pathologic tearfulness, we administered the Center for Neurologic Study-Lability Scale (CNS-LS), ${ }^{29}$ a 7-item questionnaire comprising 2 subscales ("labile crying" and "labile laughter"). A series of additional questionnaires were administered to examine the relationship of patients' pathologic tearfulness with (1) anxiety and depression (Hospital Anxiety and Depression Scale [HADS]), ${ }^{30}$ (2) impulsivity (Barratt Impulsiveness Scale [BIS $]$ ), ${ }^{31}$ (3) irritability (Irritability Questionnaire [IRQ]), ${ }^{32}$ and (4) empathy (Cambridge Behaviour Scale $[\mathrm{CBS}]^{33}$; docs.autismresearchcentre.com/tests/EQ40_ScoringKey.doc). A total of $25 / 38$ patients and $29 / 57 \mathrm{HCs}$ completed and returned those self-administered questionnaires by post. Patients filled out the questionnaires together with their next of kin or family members. Patients who completed the emotion regulation questionnaires did not differ from those who did not in the following: (1) neuropsychological tests in which patients showed preserved group-level performance ${ }^{24}$ (all $p s$, $p_{\text {corr }}$ $\geq 0.340$ ); (2) tests in which patients showed group-level impairment $^{24}$ (all $p s, p_{\text {corr }} \geq 0.304$ ); (3) clinical/demographic variables (see previous section; all $p s, p_{\text {corr }} \geq 0.999$ ); (4) volumes of manually delineated MTL structures and automatically delineated subcortical structures in which there was no group-level atrophy ${ }^{24}$ (all $p s, p_{\text {corr }} \geq 0.209$ ); and (5) structural/functional brain abnormalities identified at group level $^{24}$ (all $p$ s, $p_{\text {corr }} \geq 0.260$ ).

We also assessed the relationship of patients' emotion regulation with their memory by conducting bivariate correlation analyses between memory test scores and scores on questionnaires of emotion regulation in which patients showed impairment compared with HCs.

\section{Self-report (clinical interview)}

In a complementary approach, and since the CNS-LS may not be sensitive to the symptoms described by our patients, we dichotomized the cohort according to clinical complaint at interview. The interviewer was blind to patients' responses in the above questionnaires. Patients and their family members were asked whether there had been instances of "labile laughter" or labile crying, and to provide examples from their daily life.

\section{Relationship with demographic, clinical, and neuropsychological profiles}

We conducted (1) bivariate correlations of CNS-LS scores with continuous variables and independent-samples comparisons on CNS-LS scores for binary variables across patients; and (2) comparisons among HCs, patients with, and patients without pathologic tearfulness (independent-samples comparisons for continuous variables, $\chi^{2}$ tests for binary variables).

\section{Brain imaging}

\section{Structural MRI}

We acquired 3D T1-weighted images using a magnetizationprepared rapid gradient echo sequence (echo time $4.7 \mathrm{~ms}$, repetition time $2,040 \mathrm{~ms}, 8^{\circ}$ flip angle, field of view $192 \mathrm{~mm}$, voxel size $1 \times 1 \times 1 \mathrm{~mm}$ ). All 38 patients ( 26 male, 12 female; age at imaging: median 63.06; IQR 16.06 years) underwent structural brain imaging, along with $67 \mathrm{HCs}$ ( 35 recruited by 
the Memory and Amnesia Project [MAP]; 32 datasets were made available through OPTIMA; 40 male, 27 female; age at imaging: median 64.70; IQR 19.87 years; HCs vs patients: M: F ratio: $\chi^{2}=0.79, p=0.374$; age at imaging: $U=1,239.5$; $p=0.825)$ (methods also in reference 24 ).

\section{Volumetry}

MTL structures (left/right hippocampus, amygdala, temporopolar, entorhinal, perirhinal, and parahippocampal cortices) were manually delineated in native space (protocol: ndcn.ox. ac.uk/files/research/segmentation_protocol_medial_temporal_lobes.pdf). ${ }^{23,24}$ Subcortical structures (brainstem, left/ right thalamus, caudate nucleus, putamen, pallidum, nucleus accumbens) were automatically delineated using FSL-FIRST (v.6.0; https://fsl.fmrib.ox.ac.uk/fsl/fslwiki). ${ }^{34}$

\section{Whole-brain voxel-based morphometry (VBM)}

In order to identify gray matter (GM) volume reduction in our patient group at a whole-brain level, the T1-weighted MRIs were analyzed with VBM, conducted using Statistical Parametric Mapping software (SPM12; fil.ion.ucl.ac.uk/ $\mathrm{spm} /$ software/spm12) in MATLAB R2017b. Images were examined for scanner artefacts and reoriented to have the same point of origin (anterior commissure) and spatial orientation. They were then bias-corrected to remove intensity nonuniformities, and segmented into GM, white matter (WM), and CSF with the unified segmentation procedure. The diffeomorphic anatomical registration through the exponentiated lie algebra (DARTEL) toolbox was applied to participants' GM, WM, and CSF to refine intersubject registration, and study-specific GM templates were generated. $^{35}$ After affine registration of the GM DARTEL templates to the tissue probability maps in Montreal Neurologic Institute (MNI) space, nonlinear warping of GM images was performed to this template in MNI space. Voxel values in the tissue maps were modulated by the Jacobian determinant (calculated during spatial normalization), with modulated GM images reflecting tissue volume. These images (voxel size: $1 \mathrm{~mm}^{3}$ isotropic) were smoothed using a Gaussian filter of $8 \mathrm{~mm}$ full width at half maximum (FWHM). We compared GM volume between groups (HCs > patients; between-subject covariates: age, sex, total intracranial volume $[\mathrm{TIV}]$, study [MAP, OPTIMA]). We report clusters surviving family-wise error (FWE) correction $(p<0.05)$ at peak voxel level over $p<0.001$ (uncorrected), as well as clusters surviving correction for nonstationary smoothness ${ }^{36}$ and FWE correction for cluster size $(p<0.05)$.

Volumes (calculated from manual/automated segmentation, or the volume reflected by each VBM cluster) that showed reduction in patients at whole-group level were residualized against age, sex, TIV, and study and entered in bivariate correlation analyses with scores in questionnaires of emotion regulation. We also contrasted patients with pathologic tearfulness with those without and HCs across all volumes delineated as well as across the whole brain (VBM).

\section{Resting-state fMRI (rsfMRI)}

Whole-brain blood oxygenation level-dependent (BOLD)weighted fMRI data were acquired (gradient echo echoplanar imaging (EPI) sequence; 180 volumes; slice thickness $3.5 \mathrm{~mm}$, echo time $28 \mathrm{~ms}$, repetition time $2,410 \mathrm{~ms}, 89^{\circ}$ flip angle, field of view $192 \mathrm{~mm}$, voxel size $3 \times 3 \times 3.5 \mathrm{~mm}$ ). Participants were instructed to lie still, not to fall asleep, to keep their eyes open, and to watch a fixation cross presented on the in-scanner projector. A total of 35 of 38 patients ( 3 datasets discarded due to acquisition errors or movement; 24 men, 11 women; median age at imaging, 61.45; IQR 15.85 years) underwent rsfMRI, along with $32 \mathrm{HCs}$ (3 datasets discarded due to movement or acquisition errors; only structural MRIs were available for the HCs that were made available through OPTIMA; 23 men, 9 women; median age 55.71; IQR 17.18 years; HCs vs patients: male:female ratio: $\chi^{2}$ $=0.087 ; p=0.768$; age at imaging: $U=425.00 ; p=0.091$ ).

\section{Preprocessing}

EPIs were spatially realigned and slice time-corrected. Structural MRIs were coregistered to the EPIs, segmented and normalized along with EPIs in MNI space, followed by motion outlier detection (artifact detection tools-based scrubbing). Denoising, including the anatomical componentbased correction method (CompCor), was employed to remove sources of noise in the BOLD time series data, deriving principal components from WM and CSF. WM, $\mathrm{CSF}$, and the 6 movement measures were included as firstlevel nuisance covariates. A temporal bandpass filter (0.01-0.1 $\mathrm{Hz}$ ) was applied to this residual BOLD signal, in order to remove motion artefacts and physiologic and other artefactual effects. Images were smoothed using a Gaussian filter (8 mm FWHM).

\section{Resting-state amplitude of low frequency fluctuations and functional connectivity}

We further examined whether resting-state abnormalities in the local amplitude of low-frequency fluctuations (rsALFF) and hippocampal functional connectivity ( $\mathrm{rsFC}$ ) were associated with pathologic tearfulness. Preprocessing, rsALFF, and rsFC analyses were conducted using the CONN toolbox v. 18.a (nitrc.org/projects/conn). ${ }^{37}$

\section{rsFC: connectome-multivariate pattern analysis (MVPA)}

In order to identify seed regions for post hoc seed-to-voxel connectivity analyses in a data-driven fashion, we used MVPA as implemented in the connectome-MVPA CONN toolbox. MVPA assesses the multivariate pattern of pairwise connections between voxels across the entire brain by means of a principal component analysis (PCA) separately for each voxel that characterizes its rsFC with the rest of the brain. In the first PCA step, separately for each participant, a default number of 64 PCA components were retained while characterizing each participant's voxel-to-voxel correlation structure. The resulting component scores were stored as first-level voxel-to-voxel covariance matrices for each participant. In the 
second PCA step, separately for each voxel and jointly across participants, the 7 strongest components were retained from a PCA decomposition of the between-subjects variability in seed-to-voxel connectivity maps between this voxel and the rest of the brain, according to a conventionally employed ratio of 1:10 between the number of components extracted and the number of participants $(n=67)$. Second-level analyses were then conducted in order to test for group differences in wholebrain connectivity ( $F$ test across all MVPA components), comparing for each voxel the component scores between the 2 groups ( $\mathrm{HCs}<>$ patients; between-subjects covariates: age, sex). The results for each voxel reflected between-group differences in rsFC between this voxel and the rest of the brain.

\section{rsFC: seed-to-voxel connectivity analysis}

We followed up the MVPA with post hoc analyses to determine specific connectivity patterns. We thus conducted a whole-brain seed-to-voxel analysis, seeding from the regions identified from the MVPA contrast (HCs $<>$ patients), in order to assess connectivity between those regions and the rest of the brain.

\section{Resting-state hemodynamic activity: rsALFF}

Along with rsFC, we also examined local abnormalities in the intensity of slow spontaneous fluctuations of hemodynamic activity at rest across the whole brain, using an analysis of rsALFF, that is, the total power within the frequency range between 0.01 and $0.1 \mathrm{~Hz}$, indexing the strength of lowfrequency oscillations.

All the rsfMRI analyses involved age and sex as betweensubjects covariates. Statistical parametrical connectivity maps were thresholded at a voxel level of $p<0.001$ and FWEcorrected $(p<0.05)$ at cluster or peak level.

The mean values in clusters of reduced rsALFF or rsFC in patients at whole-group level, as compared with HCs, were residualized against age and sex across participants and then entered in bivariate correlations with scores in questionnaires of emotion regulation. We also contrasted patients with pathologic tearfulness against the rest of the patients and HCs across the whole brain.

\section{Statistical analysis}

Statistical (nonimaging) analyses were conducted using SPSS (v. 25.0, SPSS Inc., Chicago, IL). Significance values were corrected for multiple testing with the Holm-Bonferroni sequential correction method $\left(p_{\text {corr }}\right)$. We used the Levene test to assess variance homogeneity and the Shapiro-Wilk test to assess normal distribution. When normal distribution was violated (and log-transformation did not suffice), nonparametric tests were employed. Parametric (Student or Welch $t$ tests) and nonparametric tests (Mann-Whitney $U$ ) were used appropriately for independent-samples comparisons. For comparisons among 3 groups, univariate analyses of variance (ANOVAs) or Kruskal-Wallis $H$ tests were used appropriately, and post hoc comparisons between groups were Bonferroni-corrected. Pearson $r$ and Spearman $\rho$ were used appropriately to examine correlations between questionnaire scores and other measures of interest. We used multiple stepwise linear regression analysis (default $\alpha$ level of 0.05 for entry to model and 0.1 for removal) to assess the proportion of the variance of patients' scores (questionnaires on emotion regulation) that could be explained by brain abnormalities.

\section{Data availability}

The deidentified data will be available and shared by request for purposes of replicating procedures and results.

\section{Results}

\section{Emotion regulation assessment}

\section{Questionnaires: patients vs HCs}

Patients scored higher than HCs for labile crying (CNS-LS) $\left(t=-2.79, p_{\text {corr }}=0.049\right)$ but not for laughter $(t=0.44$, $p_{\text {corr }}>0.999$; 2-way mixed-effects ANOVA: group: $F=2.49, p$ $=0.12$; emotion: $F=1.81, p=0.19$; group $\times$ emotion: $F=5.73, p=0.02)$. They did not differ from HCs in their empathy quotient (CBS) $\left(t=0.79, p_{\text {corr }}>0.999\right)$, in irritability (IRQ) (frequency: $U=235, p_{\text {corr }}=0.450$; intensity: $U=240.5, p_{\text {corr }}=0.450$ ), or anxiety (HADS: $U=517$, $\left.p_{\text {corr }}=0.090\right)$. They scored higher in the planning $(t=-4.97$, $\left.p_{\text {corr }}<0.0005\right)$ and attention facets $\left(t=-3.90, p_{\text {corr }}=0.002\right)$, but not in the motor facet for impulsiveness (BIS) $(t=0.38$, $\left.p_{\text {corr }}>0.999\right)$. They also scored higher for depression (HADS) $\left(U=357.5, p_{\text {corr }}<0.0005\right)$, although no patient scored within the severe range (also noted in reference 24 ).

Scores for labile crying did not correlate across patients with impulsiveness (attention and planning facets: $\rho=0.12$, $p=0.60)$, depression $(\rho=0.24, p=0.28)$, or any memory score in which patients had shown impaired performance as compared with HCs (all $p s, p_{\text {corr }} \geq 0.240$ ), and were not associated with any demographic or clinical variables examined (all $p s, p_{\text {corr }} \geq 0.440$ ).

\section{Self-report: patients with vs patients without pathologic tearfulness and HCs}

In a research-oriented clinical interview, 19 of 38 patients were identified as presenting with pathologic tearfulness. In particular, they reported being moved to tears easily by relatively minor stimuli in a manner at odds with their premorbid state (table 1). The other 19 reported never having experienced such instances. No patient reported experiencing episodes of labile laughter.

The majority of patients and their family members reported specific triggers of such reactions, including sad stories on the news and witnessing other people crying (table 2).

Patients with pathologic tearfulness did not differ from the rest of the participants in any demographic or clinical details 
Table 1 Patients' self-reports

Pathologic tearfulness

"Since diagnosis I feel far more emotional within myself."

"I've turned into a bit of a wimp. Overwhelmed by emotion."

Triggers of pathologic tearfulness

"The news or other information [or] stories with which I have no personal connection."

"Articles on the radio [or] TV, newspapers, situations that I don't have control over."

"Children being successful [...], overcoming [...] handicap."

"When my cat brings me a 'present' e.g., a mouse, [a] bird."

Examples of the self-reports of pathologic tearfulness and triggers of pathologic tearfulness that patients with autoimmune limbic encephalitis provided to a neurologist (C.R.B.) during their research-oriented clinical interview postacutely. Each line represents a different patient's perspective.

or in episodic memory impairment, depression, or impulsiveness. Moreover, they did not differ from the rest of the patients or HCs in premorbid intelligence, vocabulary, semantic knowledge, visuomotor function or executive function, anxiety, empathy, or irritability. Among all the tests and questionnaires administered, the only one in which they scored differently from both the rest of the patients and $\mathrm{HCs}$ was CNS-LS (table 3).

Table 2 Triggers of pathologic tearfulness as identified by patients and their family members

\begin{tabular}{|c|c|c|c|c|c|c|c|c|}
\hline $\begin{array}{l}\text { Patient code } \\
\text { (see } \\
\text { reference } 24 \\
\text { for further } \\
\text { details) }\end{array}$ & $\begin{array}{l}\text { Sad stories on } \\
\text { television/ } \\
\text { newspaper/radio } \\
\text { (e.g., children } \\
\text { suffering) }\end{array}$ & Music & $\begin{array}{l}\text { Animals } \\
\text { (suffering or } \\
\text { acting } \\
\text { affectionately) }\end{array}$ & $\begin{array}{l}\text { Other } \\
\text { people } \\
\text { crying }\end{array}$ & $\begin{array}{l}\text { tamily members } \\
\text { (e.g., death, } \\
\text { departure, } \\
\text { progress, } \\
\text { overcoming } \\
\text { hardship) }\end{array}$ & $\begin{array}{l}\text { Unfamiliar } \\
\text { environment }\end{array}$ & $\begin{array}{l}\text { Photographs } \\
\text { of marriage }\end{array}$ & $\begin{array}{l}\text { No mention } \\
\text { of trigger }\end{array}$ \\
\hline 1 & & & & & & & & + \\
\hline 2 & & & & & + & & & \\
\hline 5 & & & & & & & & + \\
\hline 9 & + & & + & & & & & \\
\hline 10 & + & & & & & & & \\
\hline 11 & + & & & & & & & \\
\hline 12 & + & & & & & & & \\
\hline 13 & + & & & & & & & \\
\hline 14 & + & + & & & & & & \\
\hline 15 & + & & & + & & & & \\
\hline 17 & & & & & & + & + & \\
\hline 25 & + & & & & & & & \\
\hline 26 & & & + & & + & & & \\
\hline 27 & & & + & & & & & \\
\hline 29 & & & & & & & & + \\
\hline 30 & & & & & & & & + \\
\hline 33 & & & & & + & & & \\
\hline 34 & & & & & & & & + \\
\hline 38 & + & + & & & & & & \\
\hline
\end{tabular}


Table 3 Neuropsychological, clinical, and demographic profile of patients with vs patients without pathologic tearfulness vs healthy controls (HCs)

\begin{tabular}{|c|c|c|c|c|c|c|c|c|c|c|c|c|c|c|}
\hline \multirow[b]{2}{*}{ Domain } & \multirow[b]{2}{*}{ Test } & \multirow[b]{2}{*}{$\begin{array}{l}\text { Subtest (max } \\
\text { score/z) }\end{array}$} & \multicolumn{2}{|l|}{ HCs } & \multicolumn{2}{|c|}{ Tearful- } & \multicolumn{2}{|c|}{ Tearful+ } & \multicolumn{3}{|c|}{$\begin{array}{l}\text { Comparison among } \\
\text { groups }\end{array}$} & \multicolumn{3}{|c|}{ Post hoc comparisons } \\
\hline & & & M & IQR & M & IQR & M & IQR & Value & & $p_{\text {corr }}{ }^{a}$ & $\begin{array}{l}\text { HCs vs } \\
\text { tearful- }\end{array}$ & $\begin{array}{l}\text { Tearful+ vs } \\
\text { HCs }\end{array}$ & $\begin{array}{l}\text { Tearful+ vs } \\
\text { tearful- }\end{array}$ \\
\hline \multicolumn{15}{|c|}{ Neuropsychological profile (tearful vs nontearful patients vs HCs) } \\
\hline \multirow[t]{10}{*}{ Mood, personality, and emotion } & CNS-LS & "Labile crying" (15) & 4.0 & 2.0 & 4.0 & 4.0 & 7.0 & 4.0 & $\mathrm{~F}$ & 9.19 & 0.016 & $>0.999$ & $<0.0005$ & 0.011 \\
\hline & & "Labile laughter" (20) & 6.0 & 5.0 & 5.5 & 5.0 & 5.0 & 4.0 & $\mathrm{~F}$ & 0.15 & $>0.999$ & & & \\
\hline & BIS & Planning (44) & 20.0 & 6.0 & 26.0 & 7.3 & 26.0 & 7.0 & $\mathrm{~F}$ & 12.76 & 0.002 & $<0.0005$ & 0.003 & $>0.999$ \\
\hline & & Attention (32) & 13.0 & 3.5 & 17.5 & 8.3 & 15.0 & 1.9 & $\mathrm{~F}$ & 8.40 & 0.020 & 0.001 & 0.049 & 0.711 \\
\hline & & Motor (44) & 21.0 & 4.5 & 21.5 & 8.1 & 19.5 & 4.5 & $\mathrm{~F}$ & 1.09 & $>0.999$ & & & \\
\hline & HADS & Depression (21) & 1.0 & 2.3 & 3.0 & 8.0 & 2.0 & 4.0 & $\mathrm{H}$ & 16.69 & 0.019 & 0.015 & 0.001 & $>0.999$ \\
\hline & & Anxiety (21) & 4.0 & 4.0 & 4.5 & 7.0 & 7.0 & 2.0 & $\mathrm{H}$ & 7.80 & 0.340 & & & \\
\hline & IRQ & Frequency (63) & 13.5 & 9.0 & 15.0 & 18.5 & 17.0 & 8.8 & $\mathrm{H}$ & 3.40 & $>0.999$ & & & \\
\hline & & Intensity (63) & 17.5 & 11.3 & 17.0 & 20.8 & 21.0 & 12.0 & $\mathrm{H}$ & 2.60 & $>0.999$ & & & \\
\hline & CBS EQ (80) & & 45.0 & 11.5 & 43.5 & 14.4 & 42.4 & 25.3 & $\mathrm{~F}$ & 0.33 & $>0.999$ & & & \\
\hline \multirow{7}{*}{$\begin{array}{l}\text { Intelligence, semantic memory, } \\
\text { and language }\end{array}$} & GNT (z) & & 0.8 & 1.1 & 0.4 & 2.4 & -0.1 & 1.0 & $\mathrm{H}$ & 10.74 & 0.110 & & & \\
\hline & WASI/WASIIII & Vocabulary (z) & 1.4 & 1.3 & 0.4 & 1.6 & 0.9 & 1.5 & $\mathrm{~F}$ & 6.43 & 0.072 & & & \\
\hline & NART & p-FSIQ (z) & 1.5 & 0.8 & 0.9 & 1.2 & 1.1 & 0.8 & $\mathrm{H}$ & 8.41 & 0.285 & & & \\
\hline & WASI/WASI-II & Similarities (z) & 1.1 & 0.8 & 0.7 & 1.1 & 0.9 & 0.7 & $\mathrm{H}$ & 7.13 & 0.392 & & & \\
\hline & $C \& C T(z)$ & & 0.3 & 1.2 & 0.0 & 1.7 & 0.0 & 1.0 & $\mathrm{H}$ & 5.57 & 0.806 & & & \\
\hline & $\begin{array}{l}\text { AMI Personal } \\
\text { Semantics }\end{array}$ & $\begin{array}{l}\text { Remote: childhood } \\
\text { (21) }\end{array}$ & 19.5 & 3.0 & 18.0 & 4.1 & 16.5 & 5.0 & $\mathrm{H}$ & 7.43 & 0.360 & & & \\
\hline & & $\begin{array}{l}\text { Remote: early } \\
\text { adulthood (21) }\end{array}$ & 20.5 & 2.0 & 20.5 & 3.3 & 19.0 & 2.0 & $\mathrm{H}$ & 10.48 & 0.110 & & & \\
\hline \multicolumn{15}{|l|}{ Episodic memory } \\
\hline \multirow[t]{2}{*}{ Autobiographical } & $\begin{array}{l}\text { AMI } \\
\text { Autobiographical }\end{array}$ & $\begin{array}{l}\text { Remote: childhood } \\
\text { (9) }\end{array}$ & 9.0 & 3.0 & 5.0 & 4.8 & 4.0 & 4.0 & $\begin{array}{l}\mathrm{H} \\
17.34\end{array}$ & 0.019 & 0.001 & 0.002 & $>0.999$ & \\
\hline & & $\begin{array}{l}\text { Remote: early } \\
\text { adulthood (9) }\end{array}$ & 9.0 & 1.5 & 5.5 & 6.0 & 4.0 & 4.0 & $\begin{array}{l}\mathrm{H} \\
24.81\end{array}$ & 0.019 & 0.001 & 0.0005 & $>0.999$ & \\
\hline
\end{tabular}


Table 3 Neuropsychological, clinical, and demographic profile of patients with vs patients without pathologic tearfulness vs healthy controls (HCs) (continued)

\begin{tabular}{|c|c|c|c|c|c|c|c|c|c|c|c|c|c|c|}
\hline \multirow[b]{2}{*}{ Domain } & \multirow[b]{2}{*}{ Test } & \multirow[b]{2}{*}{$\begin{array}{l}\text { Subtest (max } \\
\text { score } / z \text { ) }\end{array}$} & \multicolumn{2}{|l|}{ HCs } & \multicolumn{2}{|c|}{ Tearful- } & \multicolumn{2}{|c|}{ Tearful+ } & \multicolumn{3}{|c|}{$\begin{array}{l}\text { Comparison among } \\
\text { groups }\end{array}$} & \multicolumn{3}{|c|}{ Post hoc comparisons } \\
\hline & & & M & IQR & M & IQR & M & IQR & Value & & $p_{\text {corr }}{ }^{a}$ & $\begin{array}{l}\text { HCs vs } \\
\text { tearful- }\end{array}$ & $\begin{array}{l}\text { Tearful+ vs } \\
\text { HCs }\end{array}$ & $\begin{array}{l}\text { Tearful+ vs } \\
\text { tearful- }\end{array}$ \\
\hline \multicolumn{15}{|c|}{ Neuropsychological profile (tearful vs nontearful patients vs HCs) } \\
\hline \multirow[t]{5}{*}{ Verbal recall } & WMS-III & Logical Memory I (z) & 0.3 & 1.9 & -1.3 & 1.1 & -1.0 & 1.7 & $\mathrm{~F}$ & 24.34 & $<0.0005$ & $<0.0005$ & $<0.0005$ & 0.464 \\
\hline & & Logical Memory II (z) & 0.7 & 1.7 & -2.5 & 1.3 & -0.7 & 2.0 & $\mathrm{H}$ & 43.48 & 0.019 & $<0.0005$ & $<0.0005$ & 0.674 \\
\hline & & Word List I (z) & 0.7 & 1.8 & -1.2 & 1.3 & -0.7 & 1.3 & $\mathrm{H}$ & 36.44 & 0.019 & $<0.0005$ & 0.001 & 0.201 \\
\hline & & Word List II (z) & 1.3 & 1.0 & -1.0 & 1.5 & -0.7 & 2.3 & $\mathrm{H}$ & 32.68 & 0.019 & $<0.0005$ & $<0.0005$ & $>0.999$ \\
\hline & $D \& P$ & People (z) & -0.3 & 1.6 & -1.3 & 1.0 & -1.3 & 1.2 & $\mathrm{H}$ & 20.40 & 0.019 & $<0.0005$ & 0.002 & $>0.999$ \\
\hline \multirow[t]{3}{*}{ Verbal recognition } & WMS-III & $\begin{array}{l}\text { Word List II } \\
\text { Recognition (z) }\end{array}$ & 0.7 & 1.0 & -1.7 & 2.4 & 0.3 & 1.7 & $\mathrm{H}$ & 31.09 & 0.019 & $<0.0005$ & 0.14 & 0.007 \\
\hline & RMT & Words (z) & 1.0 & 1.5 & -0.3 & 2.2 & 0.3 & 2.0 & $\mathrm{H}$ & 19.13 & 0.019 & $<0.0005$ & 0.004 & $>0.999$ \\
\hline & $D \& P$ & Names $(z)$ & 0.3 & 2.0 & -0.8 & 2.3 & -1.2 & 1.6 & $\mathrm{~F}$ & 13.88 & $<0.0005$ & 0.002 & $<0.0005$ & 0.872 \\
\hline \multirow[t]{3}{*}{ Visual recall } & ROCFT & Immediate Recall (z) & 1.3 & 1.9 & -1.4 & 2.9 & 0.0 & 2.6 & $\mathrm{H}$ & 26.36 & 0.019 & $<0.0005$ & 0.013 & 0.29 \\
\hline & & Delayed Recall (z) & 1.3 & 2.0 & -2.2 & 2.8 & -0.4 & 3.5 & $\mathrm{H}$ & 24.76 & 0.019 & $<0.0005$ & 0.013 & 0.385 \\
\hline & $D \& P$ & Shapes (z) & 0.7 & 1.0 & -1.0 & 2.5 & -0.7 & 2.2 & $\mathrm{~F}$ & 18.31 & $<0.0005$ & $<0.0005$ & $<0.0005$ & $>0.999$ \\
\hline \multirow[t]{3}{*}{ Visual recognition } & $D \& P$ & Doors (z) & 0.7 & 1.3 & -1.0 & 1.3 & -0.2 & 1.5 & $\mathrm{H}$ & 14.77 & 0.025 & 0.001 & 0.166 & 0.443 \\
\hline & RMT & Scenes (z) & 1.0 & 1.0 & -0.1 & 2.4 & -0.6 & 3.0 & $\mathrm{~F}$ & 12.50 & 0.001 & 0.0004 & 0.0005 & $>0.999$ \\
\hline & & Faces $(z)$ & 0.0 & 2.3 & -0.5 & 1.7 & -0.3 & 2.4 & $\mathrm{~F}$ & 0.80 & $>0.999$ & & & \\
\hline Verbal forgetting & $D \& P$ & Verbal Forgetting ( $z$ ) & 0.7 & 1.0 & -0.3 & 2.0 & -0.5 & 2.5 & $\mathrm{H}$ & 8.94 & 0.220 & & & \\
\hline Visual forgetting & & Visual Forgetting (z) & 0.3 & 0.0 & 0.3 & 2.3 & 0.3 & 1.7 & $\mathrm{H}$ & 11.42 & 0.072 & & & \\
\hline \multirow[t]{2}{*}{ Executive function } & DKEFS Trails & $\begin{array}{l}\text { Number-Letter } \\
\text { Switching }(z)\end{array}$ & 0.7 & 0.7 & 0.3 & 1.8 & 0.3 & 0.7 & $\mathrm{H}$ & 7.59 & 0.352 & & & \\
\hline & WMS-III & Digit Span (z) & 1.0 & 1.2 & 0.0 & 1.7 & 0.3 & 1.4 & $\mathrm{~F}$ & 4.42 & 0.285 & & & \\
\hline \multirow[t]{3}{*}{ Visuomotor function } & ROCFT & Copy (\%iles) & $\begin{array}{l}>16 \text { th } \\
\% \text { ile }\end{array}$ & - & $\begin{array}{l}>16 \text { th } \\
\text { \%ile }\end{array}$ & - & $\begin{array}{l}>16 \text { th } \\
\text { \%ile }\end{array}$ & - & $\mathrm{H}$ & 3.60 & $>0.999$ & & & \\
\hline & DKEFS Trails & Visual Scanning (z) & 0.7 & 1.5 & 0.3 & 1.3 & 0.0 & 1.3 & $\mathrm{H}$ & 2.48 & $>0.999$ & & & \\
\hline & & Motor Speed (z) & 0.7 & 1.0 & 0.3 & 1.3 & 0.3 & 2.7 & $\mathrm{H}$ & 5.22 & 0.876 & & & \\
\hline
\end{tabular}


Table 3 Neuropsychological, clinical, and demographic profile of patients with vs patients without pathologic tearfulness vs healthy controls (HCs) (continued)

\begin{tabular}{|c|c|c|c|c|c|c|c|c|c|c|c|c|c|}
\hline \multirow{3}{*}{$\begin{array}{l}\text { Domain } \\
\text { Neuropsychological profile (tearful vs nontearf }\end{array}$} & \multirow[b]{2}{*}{$\begin{array}{l}\text { Subtest (max } \\
\text { score/z) }\end{array}$} & \multicolumn{2}{|l|}{ HCs } & \multicolumn{2}{|c|}{ Tearful- } & \multicolumn{2}{|c|}{ Tearful+ } & \multicolumn{3}{|c|}{$\begin{array}{l}\text { Comparison among } \\
\text { groups }\end{array}$} & \multicolumn{3}{|c|}{ Post hoc comparisons } \\
\hline & & M & IQR & M & IQR & M & IQR & \multicolumn{2}{|l|}{ Value } & $p_{\text {corr }}{ }^{\mathrm{a}}$ & $\begin{array}{l}\text { HCs vs } \\
\text { tearful- }\end{array}$ & $\begin{array}{l}\text { Tearful+ vs } \\
\text { HCs }\end{array}$ & $\begin{array}{l}\text { Tearful+ vs } \\
\text { tearful- }\end{array}$ \\
\hline & \multicolumn{13}{|c|}{ Neuropsychological profile (tearful vs nontearful patients vs HCs) } \\
\hline \multirow{4}{*}{ VOSP } & Cube Analysis (10) & 10.0 & 1.0 & 9.0 & 2.0 & 9.5 & 1.3 & $\mathrm{H}$ & 4.88 & 0.957 & & & \\
\hline & $\begin{array}{l}\text { Position } \\
\text { Discrimination (20) }\end{array}$ & 20.0 & 0.3 & 20.0 & 1.0 & 20.0 & 0.0 & $\mathrm{H}$ & 2.21 & $>0.999$ & & & \\
\hline & \multirow[t]{2}{*}{ Dot Counting (10) } & 10.0 & 0.0 & 10.0 & 0.0 & 10.0 & 0.0 & $\mathrm{H}$ & 1.63 & $>0.999$ & & & \\
\hline & & \multicolumn{3}{|c|}{ Tearful- } & & \multicolumn{4}{|c|}{ Tearfult+ } & & \multicolumn{3}{|c|}{ Tearful+ vs - } \\
\hline \multicolumn{2}{|l|}{ Measure } & M & & \multicolumn{2}{|l|}{$\overline{\mathrm{IQR}}$} & \multicolumn{2}{|c|}{ M } & \multicolumn{3}{|c|}{$\mathrm{IQR}$} & \multicolumn{2}{|l|}{ Value } & $p_{\text {corr }}{ }^{2}$ \\
\hline \multicolumn{14}{|c|}{ Demographic and clinical profile (tearful vs nontearful patients) } \\
\hline \multicolumn{2}{|l|}{ Age at structural MRI, y } & \multicolumn{2}{|l|}{65.3} & \multicolumn{2}{|l|}{14.9} & \multicolumn{2}{|c|}{57.4} & \multicolumn{3}{|c|}{18.1} & $u$ & 163.00 & $>0.99$ \\
\hline \multicolumn{2}{|l|}{ Symptom onset to treatment onset delay, wk } & \multicolumn{2}{|l|}{12.0} & \multicolumn{2}{|l|}{31.0} & & & & 15.0 & & $u$ & 144.00 & $>0.99$ \\
\hline Age at symptom onset, $y$ & & 58.6 & & 11.1 & & & & & 20.0 & & $t$ & 0.774 & $>0.99$ \\
\hline Symptom onset to research scan delay, y & & 4.0 & & 4.0 & & & & & 7.5 & & $t$ & -1.09 & $>0.99$ \\
\hline Age at neuropsychological assessment, y & & 64.0 & & 13.0 & & & & & 18.1 & & $t$ & 0.20 & $>0.99$ \\
\hline Sex, M:F & & 13:6 & & & & & & & & & $x^{2}$ & 0.00 & $>0.99$ \\
\hline Premorbid depression & & $1+: 18-$ & & & & & & & & & $x^{2}$ & 0.36 & $>0.99$ \\
\hline Seizures & & & & & & & & & & & & & \\
\hline Recency (last seizure $>$ vs $<1$ year since resea & date) & $10>: 6<$ & & & & & & & & & $x^{2}$ & 1.45 & $>0.99$ \\
\hline Acute clinical T2 MRI abnormalities & & & & & & & & & & & & & \\
\hline HPC (+ vs -) & & $16+: 3-$ & & & & & & & & & $x^{2}$ & 1.12 & $>0.99$ \\
\hline AMG (+vs -) & & $5+: 14-$ & & & & & & & & & $x^{2}$ & 3.17 & $>0.99$ \\
\hline Acute symptoms & & & & & & & & & & & & & \\
\hline Depression (+ vs -) & & $6+: 13-$ & & & & & & & & & $x^{2}$ & 0.00 & $>0.99$ \\
\hline Anxiety (+ vs -) & & $7+: 12-$ & & & & & & & & & $x^{2}$ & 0.43 & $>0.99$ \\
\hline Agitation (+vs -) & & 7+:12- & & & & & & & & & $x^{2}$ & 1.69 & $>0.99$ \\
\hline
\end{tabular}




\begin{tabular}{|c|c|c|c|c|c|c|c|}
\hline \multirow[b]{2}{*}{ Measure } & \multicolumn{2}{|c|}{ Tearful- } & \multicolumn{2}{|c|}{ Tearful+ } & \multicolumn{3}{|c|}{ Tearful+ vs - } \\
\hline & $M$ & IQR & $M$ & IQR & Value & & $p_{\text {corr }}{ }^{a}$ \\
\hline Hallucinations & $2+: 17-$ & & $7+: 12-$ & & $x^{2}$ & 3.64 & $>0.99$ \\
\hline Obsessionality & $4+: 15-$ & & $3+: 16-$ & & $x^{2}$ & 0.18 & $>0.99$ \\
\hline \multicolumn{8}{|l|}{ Autoantibodies } \\
\hline Seropositive (+ vs -) & $13+: 6-$ & & $15+: 4-$ & & $x^{2}$ & 0.54 & $>0.99$ \\
\hline LG1 (+ vs -) & $8+: 11-$ & & $10+: 9-$ & & $x^{2}$ & 0.42 & $>0.99$ \\
\hline \multicolumn{8}{|l|}{ Immunotherapy } \\
\hline Oral (+ vs -) & $12+: 7-$ & & $17+: 2-$ & & $x^{2}$ & 3.64 & $>0.99$ \\
\hline PLEX (+ vs -) & $6+: 13-$ & & $5+: 14-$ & & $x^{2}$ & 0.13 & $>0.99$ \\
\hline IVlg (+ vs -) & $12+7-$ & & $13+: 6-$ & & $x^{2}$ & 0.12 & $>0.99$ \\
\hline \multicolumn{8}{|l|}{ Medication } \\
\hline BZD (+ vs -) & $2+: 17-$ & & $2+: 17-$ & & $x^{2}$ & 0.00 & $>0.99$ \\
\hline SSRI (+ vs -) & $6+: 13-$ & & $6+: 13-$ & & $x^{2}$ & 0.00 & $>0.99$ \\
\hline AED (+ vs -) & $14+: 5-$ & & $17+: 2-$ & & $x^{2}$ & 1.58 & $>0.99$ \\
\hline \multicolumn{8}{|c|}{ 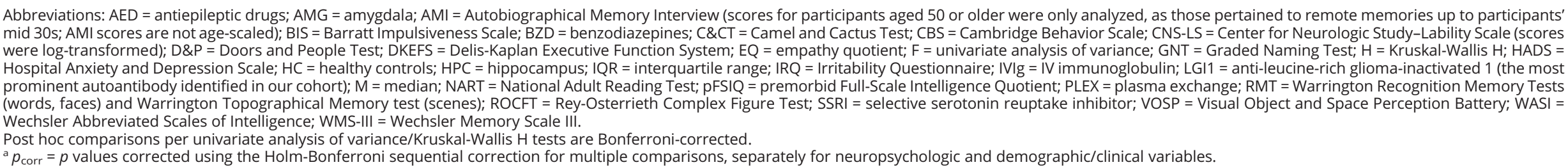 } \\
\hline
\end{tabular}




\section{Structure/function-Behavior relationships}

\section{Questionnaires: correlations with CNS-LS and BIS scores}

In our previous study, ${ }^{24}$ we identified a series of brain abnormalities $(\mathrm{n}=13)$ that patients showed at group level: volume reduction in the left and right hippocampus, captured by both VBM and manual delineation; volume reduction in the anterior-mediodorsal thalamus and right dorsolateral thalamus (VBM) and the left thalamus (automated delineation), as well as the right entorhinal cortex (manual delineation); reduced right hippocampal rsFC with left hippocampus, ventral-posterior posteromedial cortex (posterior cingulate, retrosplenial cortex, and precuneus; Brodmann area [BA] 23, 31), and medial prefrontal cortex (BA 10, 32, 24); and reduced rsALFF in the posterior cingulate and the precuneus (BA 23, 31). We entered the mean values of the clusters that reflected these abnormalities (residualized against age and sex for functional abnormalities, as well as TIV and study [MAP, OPTIMA] for volumes) in bivariate correlations with CNS-LS scores for labile crying. Patients' scores correlated strongly with their reduced right hippocampal rsFC with the ventral-posterior posteromedial cortex $(r=$ $-0.61, p_{\text {corr }}=0.030$; rest of $p s, p_{\text {corr }} \geq 0.190$; figure $\left.1 \mathrm{~A}\right)$. No such correlations were identified with impulsivity (BIS attention and planning facets) or depression (HADS) scores, even at uncorrected levels $(|r|<0.29, p>0.18)$.

Moreover, given our a priori hypotheses on the role of the hippocampus in emotion dysregulation, we examined, at uncorrected levels, correlations with CNS-LS scores. Right anterior hippocampal volume correlated negatively across patients with scores for labile crying $(r=-0.52, p=0.01$; left anterior, right/left posterior hippocampus: $p>0.07$; figure
1B), but not with right hippocampal rsFC with the posteromedial cortex $(r=0.33, p=0.05)$. When these 2 factors were entered as independent variables in a multiple stepwise linear regression, the analysis was terminated in 2 steps, with the right hippocampal-posteromedial cortical rsFC included in the first model as a predictor of patients' scores of labile crying $\left(F=12.50, p=0.002 ; R^{2}=0.37\right)$, and with the volume of the right anterior hippocampus entered in the model in the second step $\left(F=9.55, p=0.001 ; R^{2}=0.49\right)$. No volumetric correlation of any hippocampal segment was identified with impulsivity or depression scores $(|r|<0.25$, $p>0.19)$.

\section{Self-report: patients with vs without pathologic tearfulness and HCs}

\section{Structural abnormalities}

Patients with pathologic tearfulness did not differ from the rest of the patients in any MTL or subcortical volumes $\left(p_{\text {corr }} \geq\right.$ $0.350)$. Nevertheless, a whole-brain VBM analysis disclosed lower volume for these patients relative to the other 2 groups in the right anterior hippocampus, the right cerebellar hemispheric HVI/HVIIa Crus I, and the left fusiform gyrus (BA 37; figure 2).

\section{Functional abnormalities}

A connectome-MVPA analysis on rsFC across the whole brain identified a cluster in the right hippocampus as a region in which patients with pathologic tearfulness differed from the other 2 groups. We thus seeded from the right hippocampus in native space (unsmoothed timeseries), in order to identify regions with which these patients showed abnormal right hippocampal rsFC: they showed aberrantly increased right hippocampal rsFC with the right middle frontal gyrus (BA 9)

Figure 1 "Labile crying" scores in patients with autoimmune limbic encephalitis: structure/function-behavior relationships

A

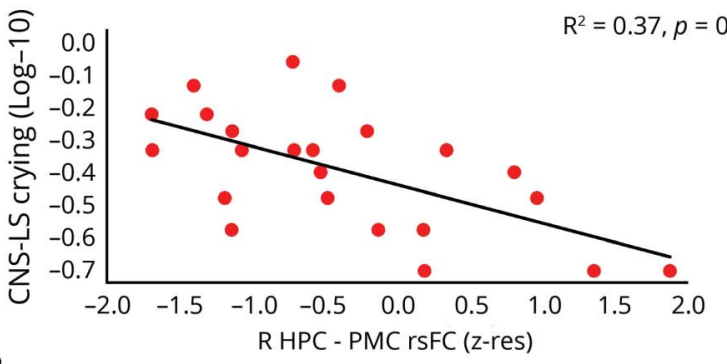

B

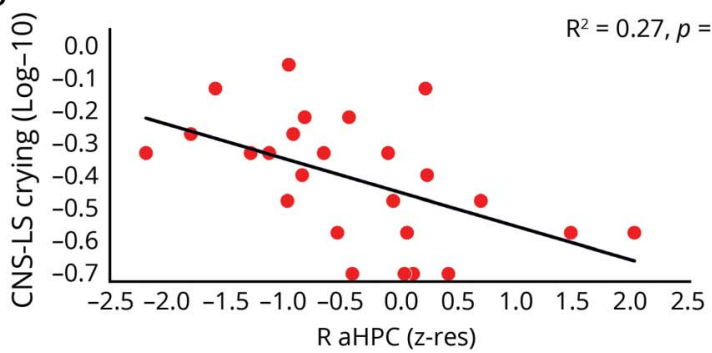

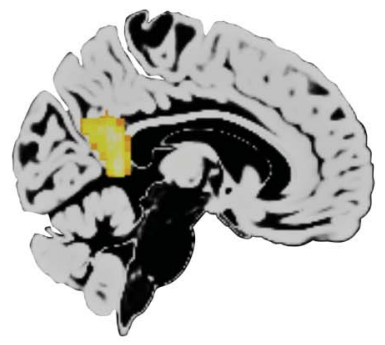

Bivariate correlations between Center for Neurologic Study-Lability Scale (CNS-LS) scores for labile crying (log-transformed) and measures of structural and functional abnormalities. (A) Correlation with mean resting-state functional connectivity (rsFC) between the right hippocampus (HPC) and posteromedial cortex (PMC). (B) Correlation with volume of the (manually delineated) right anterior $\mathrm{HPC}$ (head). Red = right $\mathrm{HPC}$ head; yellow = right HPC body; teal = right HPC tail; green = left HPC head; blue = left HPC body; pink = left HPC tail. $\mathrm{R}, \mathrm{L}=$ right, left (hemisphere); $\mathrm{z}$ res $=$ volumes are residualized against age, sex, total intracrania volume, and study (Memory and Amnesia Project, Oxford Project to Investigate Memory and Ageing) across participants; mean rsFC values are residualized against age and sex across participants. 
A

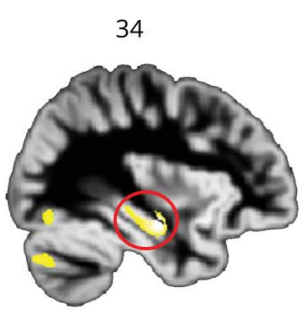

R aHPC

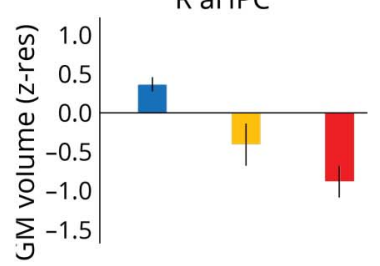

B
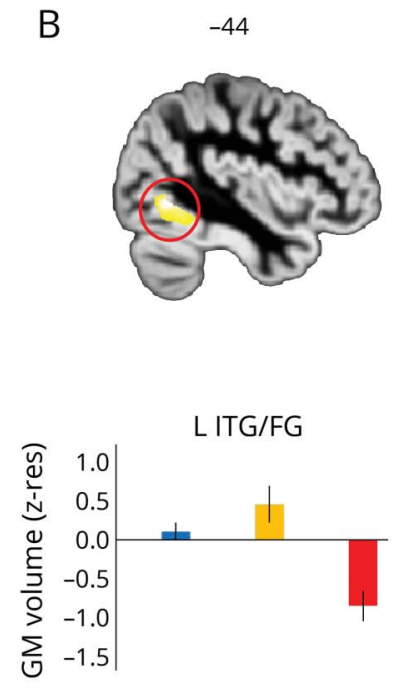

C
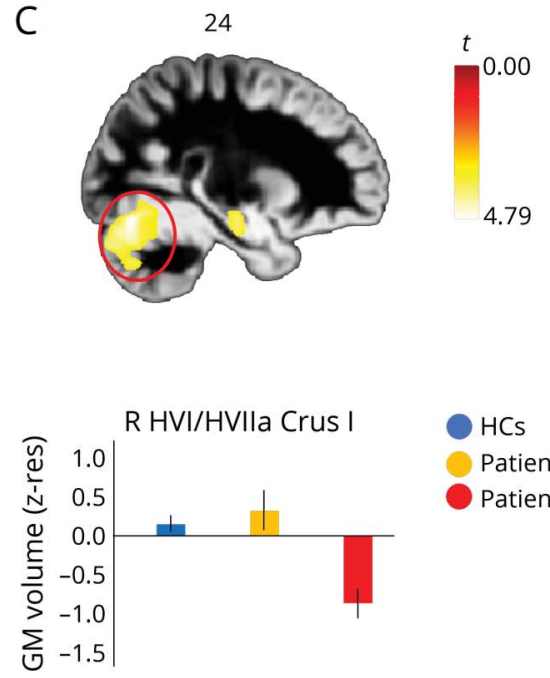

$\mathrm{HCS}$

Patients (tearful -)

Patients (tearful +)

Results of whole-brain voxel-based morphometry (VBM) on modulated gray matter (GM) (reflecting GM volume). Contrast: healthy controls (HCs) and patients without pathologic tearfulness > patients with pathologic tearfulness; between-subjects nuisance regressors: age, sex, total intracranial volume (TIV), and study (Memory and Amnesia Project [MAP], Oxford Project To Investigate Memory and Ageing [OPTIMA]). (A) Right anterior hippocampus: kE = 19, $p$ familywise error-corrected $(F W E)=0.037$; peak voxel: $t=4.79 ; x=34, y=-12, z=-17$. (B) Left fusiform gyrus/posterior portion of inferior temporal gyrus: $\mathrm{kE}=17$; $p$ $\mathrm{FWE}=0.038$; peak voxel: $t=4.79 ; \mathrm{x}=-44, \mathrm{y}=-62, \mathrm{z}=-5$. (C) Right cerebellar hemispheric lobules VI/VIIa Crus I: $\mathrm{kE}=23 ; p \mathrm{FWE}=0.042 ;$ peak voxel: $t=4.76 ; \mathrm{x}=24$, $\mathrm{y}=-75, \mathrm{z}=-18$; clusters are displayed here at $p<0.001$ (unc) for display purposes, and survive FWE correction ( $p<0.05$ ) at peak-voxel level over $p<0.001$ (unc) (minimum cluster volume: kE > 10). The cerebellar cluster also survived correction for nonstationary smoothness and cluster size ( $\mathrm{p}$-FWE < 0.05 ). Clusters are overlaid here on a diffeomorphic anatomical registration through exponentiated lie algebra GM template in Montreal Neurological Institute space (sagittal sections presented); heat bar represents $t$ values; bar graphs display the average GM volume of each of those 3 clusters for the 3 different groups; error bars represent +1/-1 SEM. aHPC = anterior hippocampus; FG = fusiform gyrus; ITG = inferior temporal gyrus; $k E=$ cluster size (number of voxels); R, L = right, left (hemisphere); z-res = mean values residualized against age, sex, study (MAP, OPTIMA), and TIV across participants.

and reduced $\mathrm{rsFC}$ with a region in the right posterior cingulate extending to the precuneus and lingual gyrus (BA 23, 18). Patients with pathologic tearfulness also showed aberrantly increased rsALFF in the left fusiform gyrus (BA 37) and the ventral pons, as well as reduced rsALFF in the right inferior parietal lobule (BA 39; figure 3).

\section{Discussion}

Our study is the first to investigate the nature and neural foundations of emotion dysregulation in a uniquely large, homogeneous cohort of patients after aLE, a nondegenerative neurologic syndrome characterized by primary limbic pathology.

\section{Clinical features and correlates of emotion dysregulation}

In particular, we describe a novel disorder of emotion regulation following aLE that is characterized by residual pathologic tearfulness. In our cohort, this was reported by $50 \%$ of patients. This symptom may be misdiagnosed as a manifestation of depression; for example, an indirect consequence of reduced quality of life due to memory impairment. If present alongside disinhibition and impulsiveness, it may otherwise be interpreted as a sign of a broader dysexecutive syndrome, continuous with that sometimes present in the acute stage of aLE. ${ }^{10}$ However, we showed that pathologic tearfulness was not associated with depression or impulsiveness, and occurred in the face of preserved executive function, and at normal levels of anxiety and irritability. Notably, no clinical or behavioral difference was detected between the patients with pathologic tearfulness and the equally sized subset with no such symptoms, apart from their scores on labile crying (CNS-LS).

To our knowledge, this symptom has only been mentioned in passing in case or case series studies of aLE ${ }^{13-15}$ or in studies of larger yet less homogeneous cohorts of autoimmune encephalitis or epilepsy, ${ }^{38,39}$ as "emotional lability," "mood lability," or "uncharacteristic tearfulness," with no further discussion of its clinical features and correlates. Direct comparisons with other patient groups such as temporal lobe epilepsy will be needed in future studies. Moreover, the profile of pathologic tearfulness observed in our patients with aLE is strikingly different from the syndrome of pseudobulbar affect seen in other neurologic conditions (e.g., amyotrophic lateral sclerosis, stroke, multiple sclerosis, Parkinson disease, Alzheimer disease, and traumatic brain injury ${ }^{1-5}$ ), where dramatic and debilitating bouts of laughing or crying occur often without any appropriately valanced trigger ${ }^{7}$ or congruence between the experience and expression of emotion. ${ }^{40}$ For instance, none of our patients presented with pathologic laughing. Furthermore, most patients who presented with pathologic tearfulness readily identified specific triggers that were congruent with their albeit exaggerated emotional 


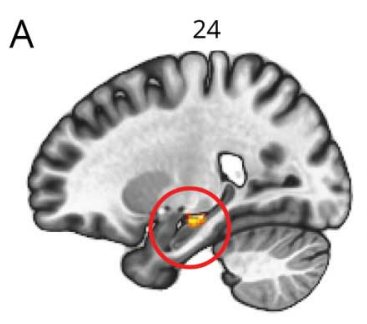

A

0
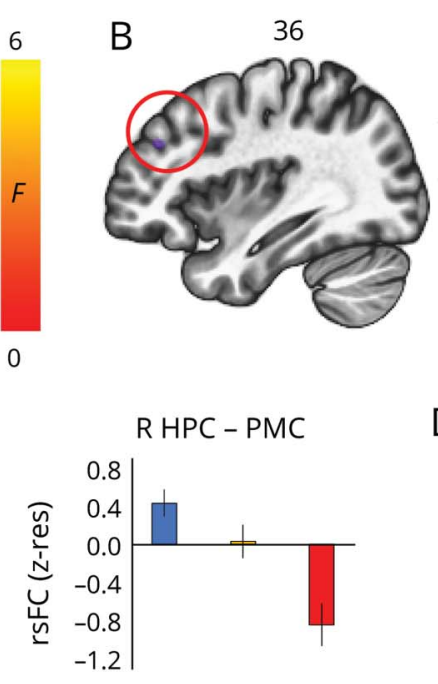

E

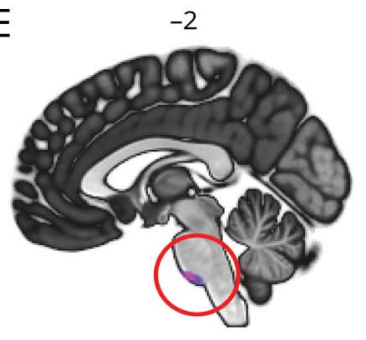

C

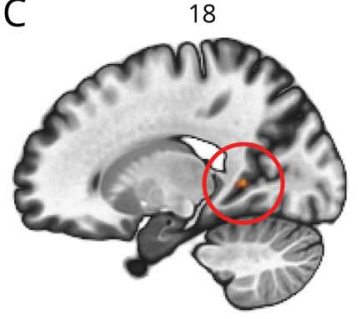
18

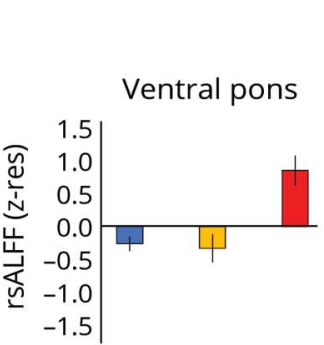

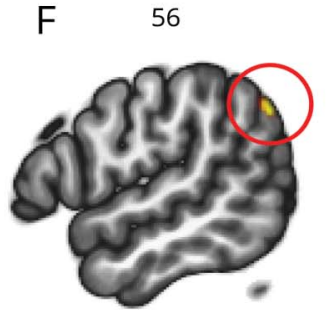

D
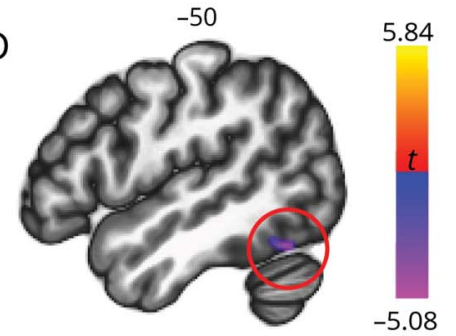

$\mathrm{HCS}$

Patients (tearful-)

Patients (tearful+)

(A) A connectome-multivariate pattern analysis demonstrated that patients with pathologic tearfulness, as compared with the rest of the patients and healthy controls (HCs), showed abnormal resting-state functional connectivity (rsFC) between a region in the right hippocampal (HPC) head and body and the rest of the brain; right hippocampal head and body: $24-16-14, \mathrm{kE}=194 ; p$ family-wise error-corrected (FWE) (cluster-level) $=0.001$. (B-C) Patients with pathologic tearfulness showed $(\mathrm{B})$ increased $\mathrm{rsFC}$ of the right hippocampus with the right middle frontal gyrus $(\mathrm{MFG})(\mathrm{x}=36, \mathrm{y}=40, \mathrm{z}=30 ; \mathrm{kE}=105, p \mathrm{FWE}=0.03, t=-4.09)$, and (C) reduced rSFC of the right hippocampus with the posteromedial cortex (PMC) (peak voxel: $t=4.65 ; x=18, y=-50, z=6 ; k E=98, p$ FWE $=0.04)$, extending to the right lingual gyrus. (D-F) Patients with pathologic tearfulness showed aberrantly increased resting-state amplitude of low frequency fluctuations (rsALFF) as compared with both the rest of the patients and healthy controls in (D) the left fusiform gyrus ( $k E=69 ;$ peak voxel: $t=-4.72 ; x=-50, y=-60, z=-20)$, and $(E)$ the ventral pons ( $k E=74$; peak voxel: $t=-5.08 ; x=-2, y=-20, z=-44 ; p$ FWE $=0.04$ ), and reduced rsALFF in (F) the right inferior parietal lobule (peak voxel: $t=5.84 ; \mathrm{x}=56, \mathrm{y}=-62, \mathrm{z}=40 ; \mathrm{kE}=112 ; p \mathrm{FWE}=0.004)$. All clusters survive FWE correction $(p<0.05)$ for cluster size over an uncorrected individual voxel threshold of $p<0.001$. Error bars represent \pm 1 SEM. FG = fusiform gyrus; IPL = inferior parietal lobule; $k E=$ cluster size; $\mathrm{R}, \mathrm{L}=$ right, left (hemisphere); $\mathrm{z}$-res = mean rsFC and rSALFF values are residualized against age and sex across participants.

responses. Many of these triggers pertained to situations that evoked empathic concern (e.g., children or animals in distress or acting affectionately). This may suggest that aberrantly increased empathy underlies the patients' symptoms. There are, indeed, strong links between empathy and proneness to crying in the healthy population, which may suggest that increased empathy is associated with increased likelihood to experience distress, resulting in a higher crying proneness (see reference 41). Whereas the CBS did not disclose abnormality in patients with pathologic tearfulness, it may lack sensitivity in capturing increased, rather than decreased, empathy. Likewise, while the CNS-LS represents the most broadly employed self-report measure of affective lability, ${ }^{29}$ more targeted instruments need to be employed, examining autonomic responses within the context of finer-grained behavioral tasks. This might lead to identification of similar symptoms in other neurologic disorders, such as temporal lobe epilepsy, where suggestive evidence has already been presented. ${ }^{42}$

\section{Structural and functional correlates}

In line with our hypotheses, we found correlates of pathologic tearfulness in the anterior hippocampus, the posterior cingulate cortex, the ventral pons, and the neocerebellum. Whether these abnormalities result directly from the acute, primary pathology of aLE or occur subsequently as a form of functional diaschisis or as a consequence of Wallerian degeneration $^{23}$ remains to be determined (see also discussion in reference 12). Figure 4 summarizes the insight our study provides on the impairments underlying pathologic tearfulness in aLE, based on the model in reference 7 .

\section{Anterior hippocampal volume}

Right anterior hippocampal atrophy was associated with pathologic tearfulness. The right anterior hippocampal volume correlated across patients with scores for labile crying (CNS-LS), and patients with pathologic tearfulness showed less volume in the right anterior hippocampus in a voxel-wise 


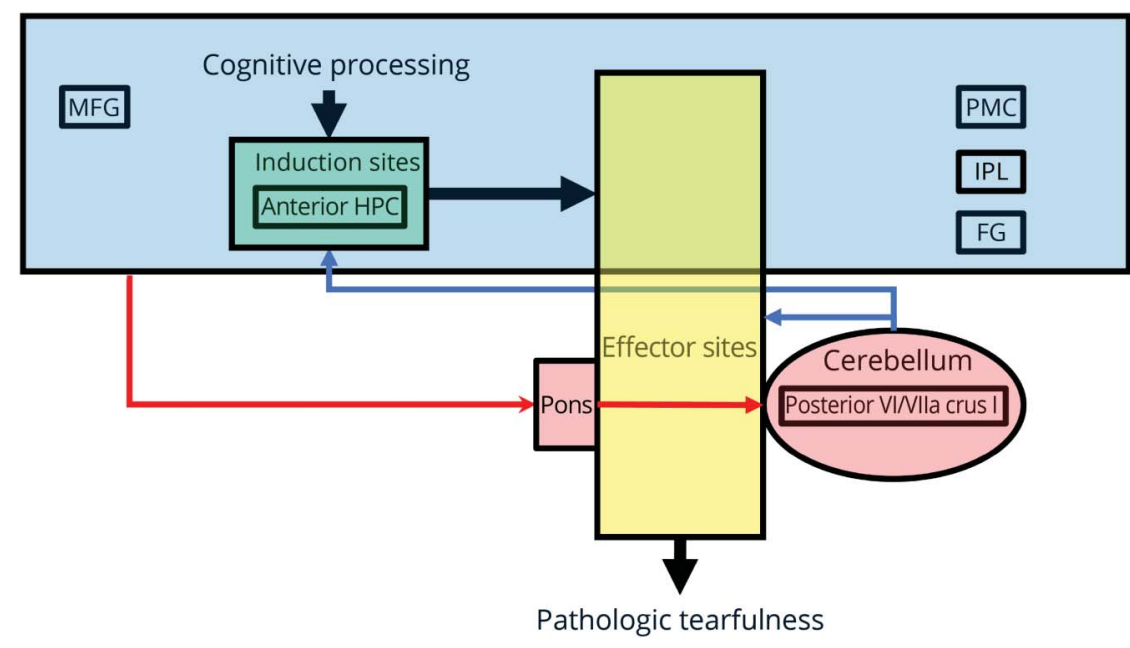

Illustration of the network abnormalities that may underlie pathologic tearfulness following aLE, based on our findings and on the model of "pathological laughing and crying" proposed by Parvizi et al. (2001). The locations mentioned in the figure are only those identified as abnormal in this study, and other regions are likely to be involved as well. Blue box = telencephalic sites that are assumed to process "emotionally competent" stimuli along with relevant context information that may include the middle frontal gyrus (MFG), the posterior ventral posteromedial cortex (PMC), the inferior parietal lobule (IPL), and the fusiform gyrus (FG); these act on the induction sites (green box) (e.g., ventromedial prefrontal cortex, anterior cingulate, amygdala, ventral striatum), which may also include the anterior hippocampus (HPC); these sites detect the stimuli and context, and act on the effector sites (yellow box) (e.g., motor cortex, hypothalamus, periaqueductal gray, cranial nerve nuclei), which trigger the emotional response. Red arrows = cerebro-ponto-cerebellar pathways, through which telencephalic areas convey to the cerebellum information on the emotionally competent stimuli along with contextrelated information; blue arrows = the cerebellum modulates the profile, intensity, and duration of the emotional responses in accordance with the context of the triggering stimulus by providing input to the induction and effector sites; structural and functional abnormalities in these sites may trigger emotional responses (pathologic tearfulness) that are contextually inappropriate.

whole-brain analysis. That hippocampal lesions should be associated with pathologic tearfulness is consistent with the involvement of limbic circuitry in emotion processing, ${ }^{17}$ especially with the relationship between recurrent stress and hippocampal damage in nonhuman primates, ${ }^{19}$ as well as with hippocampal pathology in psychiatric disorders. ${ }^{43}$ In particular, the primate anterior hippocampus is the homologue of the rodent ventral hippocampus, which plays a role in negative affect, by virtue of its connectivity with the amygdala and the hypothalamus. ${ }^{18}$ However, manually delineated hippocampal volumes did not differ between patients with and those without pathologic tearfulness, suggesting that atrophy may be confined to specific regions within the anterior hippocampus, a possibility that could be explored using subfield volumetry in future studies.

\section{Hippocampal dysconnectivity with the posteromedial cortex}

Scores for labile crying (CNS-LS) strongly correlated with patients' reduced right hippocampal rsFC with the ventral posteromedial cortex (posterior cingulate, retrosplenial cortex, and precuneus). Evidence from functional neuroimaging of healthy adults supports a role of this region in empathic concern for emotional suffering and admiring virtue. ${ }^{44} \mathrm{Ab}$ errant perspective taking and empathy has also been reported in hippocampal patients. ${ }^{45,46}$

\section{Pontocerebellar abnormalities}

Volume reduction was also noted for patients with pathologic tearfulness in posterior portions of the right hemispheric cerebellar lobules VI/VIIa Crus I. These regions are embedded within the default mode network, which is fundamental for self-referential cognition. ${ }^{47}$ The cerebellum receives input from the basilar pons, and disruption of cortico-pontocerebellar circuits may lower the threshold for emotional expression. ${ }^{7}$ Moreover, anatomical and electrophysiologic work has recently disclosed evidence for cerebellar (lobules VI/VIIa Crus I)-hippocampal interactions, possibly via the pons. ${ }^{25}$ Consistent with these accounts, patients with pathologic tearfulness demonstrated aberrantly increased rsALFF in the ventral pons. The ventral pons relays input to the cerebellum from cortical regions including the parietal association corti$\mathrm{ces}^{48}{ }^{48}$ where these patients showed reduced right hippocampal rsFC. Interestingly, pontine hemodynamic hyperactivity has been reported previously in a single case study of pathologic laughing, ${ }^{6}$ consistent with earlier reports of pathologic crying in cases of pontine myelinosis. ${ }^{8}$

\section{Abnormalities in the inferior parietal lobule, fusiform, and middle frontal gyri}

Beyond the relationships that we had hypothesized, we also observed a series of unpredicted abnormalities associated with pathologic tearfulness: GM volume reduction and aberrantly increased rsALFF in the left fusiform gyrus, reduced rsALFF in the right inferior parietal lobule, and reduced rsFC between the right hippocampus and the right middle frontal gyrus. While activations in all of these regions have been repeatedly shown in self-face processing, ${ }^{49,50}$ the aberrantly increased rsFC and rsALFF in patients with pathologic tearfulness require further investigation, as they may reflect compensatory or maladaptive mechanisms. 
Our study describes a novel disorder of emotion regulation following aLE that is characterized by residual pathologic tearfulness, is not related to low mood or cognitive impairment, and is associated with specific abnormalities within networks supporting emotion regulation. Clinicians need to be aware of the potential for such symptoms to develop after $\mathrm{aLE}$ and of the distress they can cause. Furthermore, pathologic tearfulness offers a useful neuropsychological model for exploring the neural mechanisms of emotion regulation and may provide insight into the breakdown of these mechanisms across a wide range of neurologic conditions. This will inform the development and refinement of behavioral and pharmaceutical interventions.

\section{Acknowledgment}

The authors thank the participants of this study.

\section{Study funding}

C.R.B. is supported by a Medical Research Council Clinician Scientist Fellowship (MR/K010395/1). S.R.I. is supported by the Wellcome Trust (104079/Z/14/Z), the UCB-Oxford University Alliance, BMA Research Grants-Vera Down grant (2013) and Margaret Temple (2017), Epilepsy Research UK (P1201), and the Fulbright UK-US commission (MS Society Research Award). The research was funded/supported by the National Institute for Health Research (NIHR) Oxford Biomedical Research Centre. The views expressed are those of the authors and not necessarily those of the NHS, the NIHR, or the Department of Health.

\section{Disclosure}

G. Argyropoulos, L. Moore, C. Loane, A. Roca-Fernandez, C. Lage-Martinez, and O. Gurau report no disclosures relevant to the manuscript. S. Irani is a coapplicant and receives royalties on patent application WO/2010/046716 (UK patent no. PCT/ GB2009/051441) titled "Neurologic autoimmune disorders." The patent has been licensed to Euroimmun AG for the development of assays for LGI1 and other VGKC-complex antibodies. A. Zeman and C. Butler report no disclosures relevant to the manuscript. Go to Neurology.org/ $\mathrm{N}$ for full disclosures.

\section{Publication history}

Received by Neurology July 4, 2019. Accepted in final form October 3, 2019.

Appendix Authors

\begin{tabular}{|c|c|c|c|}
\hline Name & Location & Role & Contribution \\
\hline $\begin{array}{l}\text { Georgios P.D. } \\
\text { Argyropoulos, } \\
\text { PhD }\end{array}$ & $\begin{array}{l}\text { University of } \\
\text { Oxford, UK }\end{array}$ & Author & $\begin{array}{l}\text { Study concept and } \\
\text { design, major role in } \\
\text { the acquisition of } \\
\text { data and analysis, } \\
\text { drafting the } \\
\text { manuscript and } \\
\text { preparing figures, } \\
\text { interpreted the data, } \\
\text { revised the }\end{array}$ \\
\hline
\end{tabular}

\section{Appendix (continued)}

\begin{tabular}{|c|c|c|c|}
\hline Name & Location & Role & Contribution \\
\hline & & & $\begin{array}{l}\text { manuscript for } \\
\text { intellectual content }\end{array}$ \\
\hline $\begin{array}{l}\text { Lauren Moore, } \\
\text { BSc }\end{array}$ & $\begin{array}{l}\text { University of } \\
\text { Oxford; University } \\
\text { of Bath, UK }\end{array}$ & Author & $\begin{array}{l}\text { Study concept and } \\
\text { design, major role in } \\
\text { the acquisition of } \\
\text { data }\end{array}$ \\
\hline $\begin{array}{l}\text { Clare Loane, } \\
\text { PhD }\end{array}$ & $\begin{array}{l}\text { University of } \\
\text { Oxford; King's } \\
\text { College London, UK }\end{array}$ & Author & $\begin{array}{l}\text { Study concept and } \\
\text { design, major role in } \\
\text { the acquisition of } \\
\text { data }\end{array}$ \\
\hline $\begin{array}{l}\text { Adriana Roca- } \\
\text { Fernandez, } \\
\text { MSc }\end{array}$ & $\begin{array}{l}\text { University of } \\
\text { Oxford, UK }\end{array}$ & Author & $\begin{array}{l}\text { Major role in the } \\
\text { acquisition of data }\end{array}$ \\
\hline $\begin{array}{l}\text { Carmen Lage- } \\
\text { Martinez, MD }\end{array}$ & $\begin{array}{l}\text { University of } \\
\text { Oxford, UK; } \\
\text { University Hospital } \\
\text { Marqués de } \\
\text { Valdecilla, } \\
\text { Santander, Spain }\end{array}$ & Author & $\begin{array}{l}\text { Major role in the } \\
\text { acquisition of data }\end{array}$ \\
\hline $\begin{array}{l}\text { Oana Gurau, } \\
\text { MSc }\end{array}$ & $\begin{array}{l}\text { University of } \\
\text { Oxford, UK }\end{array}$ & Author & $\begin{array}{l}\text { Major role in the } \\
\text { acquisition of data }\end{array}$ \\
\hline $\begin{array}{l}\text { Sarosh R. } \\
\text { Irani, FRCP, } \\
\text { PhD }\end{array}$ & $\begin{array}{l}\text { University of } \\
\text { Oxford, UK }\end{array}$ & Author & $\begin{array}{l}\text { Interpreted the data, } \\
\text { revised the } \\
\text { manuscript for } \\
\text { intellectual content }\end{array}$ \\
\hline $\begin{array}{l}\text { Adam Zeman, } \\
\text { FRCP }\end{array}$ & $\begin{array}{l}\text { University of } \\
\text { Exeter, UK }\end{array}$ & Author & $\begin{array}{l}\text { Interpreted the data, } \\
\text { revised the } \\
\text { manuscript for } \\
\text { intellectual content }\end{array}$ \\
\hline $\begin{array}{l}\text { Christopher R. } \\
\text { Butler, FRCP, } \\
\text { PhD }\end{array}$ & $\begin{array}{l}\text { University of } \\
\text { Oxford, UK; } \\
\text { Imperial College } \\
\text { London, London; } \\
\text { Pontificia } \\
\text { Universidad } \\
\text { Católica de Chile }\end{array}$ & Author & $\begin{array}{l}\text { Study concept and } \\
\text { design, major role in } \\
\text { the acquisition of } \\
\text { data and analysis, } \\
\text { drafting the } \\
\text { manuscript or } \\
\text { preparing figures, } \\
\text { interpreted the data, } \\
\text { revised the } \\
\text { manuscript for } \\
\text { intellectual content }\end{array}$ \\
\hline
\end{tabular}

\section{References}

1. Floeter MK, Katipally R, Kim MP, et al. Impaired corticopontocerebellar tracts un derlie pseudobulbar affect in motor neuron disorders. Neurology 2014;83:620-627.

2. Brooks BR, Thisted RA, Appel SH, et al. Treatment of pseudobulbar affect in ALS with dextromethorphan/quinidine: a randomized trial. Neurology 2004;63: 1364-1370.

3. Kim JS, Choi-Kwon S, Elkind MSV. Poststroke depression and emotional incontinence: correlation with lesion location. Neurology 2000;54:1805-1810.

4. Choi-Kwon S, Han K, Choi S, et al. Poststroke depression and emotional in continence: factors related to acute and subacute stages. Neurology 2012;78: $1130-1137$.

5. Fitzgerald KC, Salter A, Tyry T, Fox RJ, Cutter G, Marrie RA. Pseudobulbar affect. Neurol Clin Pract 2018;8:472-481.

6. Kosaka H, Omata N, Omori M, et al. Abnormal pontine activation in pathological laughing as shown by functional magnetic resonance imaging. J Neurol Neurosurg Psychiatry 2006;77:1376-1380.

7. Parvizi J, Anderson SW, Martin CO, Damasio H, Damasio AR. Pathological laughter and crying: a link to the cerebellum. Brain 2001;124:1708-1719.

8. Van Hilten JJ, Buruma OJS, Kessing P, Vlasveld LT. Pathologic crying as a prominent behavioral manifestation of central pontine myelinolysis. Arch Neurol 1988;45:936.

9. Thompson J, Bi M, Murchison AG, et al. The importance of early immunotherapy in patients with faciobrachial dystonic seizures. Brain 2018;141:348-356.

10. Butler CR, Miller TD, Kaur MS, et al. Persistent anterograde amnesia following limbic encephalitis associated with antibodies to the voltage-gated potassium channel complex. J Neurol Neurosurg Psychiatry 2014;85:387-391. 
11. Finke C, Prüss H, Heine J, et al. Evaluation of cognitive deficits and structural hippocampal damage in encephalitis with leucine-rich, glioma-inactivated 1 antibodies. JAMA Neurol 2017;74:50-59.

12. Iranzo A, Graus F, Clover L, et al. Rapid eye movement sleep behavior disorder and potassium channel antibody-associated limbic encephalitis. Ann Neurol 2006;59: $178-181$.

13. Schimmel M, Frühwald MC, Bien CG. Limbic encephalitis with LGI1 antibodies in a 14-year-old boy. Eur J Paediatr Neurol 2018;22:190-193.

14. Somers KJ, Sola CL. Voltage-gated potassium channel-complex antibody-associated limbic encephalitis. Psychosomatics 2011;52:78-81.

15. Naasan G, Irani SR, Bettcher BM, Geschwind MD, Gelfand JM. Episodic bradycardia as neurocardiac prodrome to voltage-gated potassium channel complex/leucine-rich, glioma inactivated 1 antibody encephalitis. JAMA Neurol 2014;71:1300-1304.

16. Khan NL, Jeffree MA, Good C, Macleod W, Al-Sarraj S. Histopathology of VGKC antibody-associated limbic encephalitis. Neurology 2009;72:1703-1705.

17. Papez JW. A proposed mechanism of emotion. Arch Neurol Psychiatry 1937;38:725.

18. Bannerman D, Rawlins JN, McHugh S, et al. Regional dissociations within the hippocampus: memory and anxiety. Neurosci Biobehav Rev 2004;28:273-283.

19. Sapolsky RM, Uno H, Rebert CS, Finch CE. Hippocampal damage associated with prolonged glucocorticoid exposure in primates. J Neurosci 1990;10:2897-2902.

20. Wagner J, Witt JA, Helmstaedter C, Malter MP, Weber B, Elger CE. Automated volumetry of the mesiotemporal structures in antibody-associated limbic encephalitis. J Neurol Neurosurg Psychiatry 2015;86:735-742.

21. Holtmann O, Schlossmacher I, Moenig C, et al. Amygdala enlargement and emotional responses in (autoimmune) temporal lobe epilepsy. Sci Rep 2018;8:9561.

22. Bubb EJ, Kinnavane L, Aggleton JP. Hippocampal-diencephalic-cingulate networks for memory and emotion: an anatomical guide. Brain Neurosci Adv 2017;1: 239821281772344

23. Loane C, Argyropoulos GPD, Roca-Fernández A, et al. Hippocampal network abnormalities explain amnesia after VGKCC-Ab related autoimmune limbic encephalitis. J Neurol Neurosurg Psychiatry 2019;90:965-974.

24. Argyropoulos GPD, Loane C, Roca-Fernandez A, et al. Network-wide abnormalities explain memory variability in hippocampal amnesia. Elife 2019;8:e46156.

25. Watson TC, Obiang P, Torres-Herraez A, et al. Anatomical and physiological foundations of cerebello-hippocampal interaction. Elife 2019;8:e41896.

26. Graus F, Titulaer MJ, Balu R, et al. A clinical approach to diagnosis of autoimmune encephalitis. Lancet Neurol 2016;15:391-404.

27. Graus F, Escudero D, Oleaga L, et al. Syndrome and outcome of antibody-negative limbic encephalitis. Eur J Neurol 2018;25:1011-1016.

28. Malter MP, Elger CE, Surges R. Diagnostic value of CSF findings in antibodyassociated limbic and anti-NMDAR-encephalitis. Seizure 2013;22:136-140.

29. Moore SR, Gresham LS, Bromberg MB, Kasarkis EJ, Smith RA. A self report measure of affective lability. J Neurol Neurosurg Psychiatry 1997;63:89-93.

30. Zigmond AS, Snaith RP. The Hospital Anxiety and Depression Scale. Acta Psychiatr Scand 1983;67:361-370.

31. Patton JH, Stanford MS, Barratt ES. Factor structure of the Barratt Impulsiveness Scale. J Clin Psychol 1995;51:768-774.
32. Craig KJ, Hietanen H, Markova IS, Berrios GE. The Irritability Questionnaire: a new scale for the measurement of irritability. Psychiatry Res 2008;159:367-375.

33. Baron-Cohen S, Wheelwright S. The Empathy Quotient: an investigation of adults with Asperger syndrome or high functioning autism, and normal sex differences. J Autism Dev Disord 2004;34:163-175.

34. Patenaude B, Smith SM, Kennedy DN, Jenkinson M. A Bayesian model of shape and appearance for subcortical brain segmentation. Neuroimage 2011;56:907-922.

35. Ashburner J. A fast diffeomorphic image registration algorithm. Neuroimage 2007;38: 95-113.

36. Hayasaka S, Phan KLL, Liberzon I, Worsley KJ, Nichols TE. Nonstationary clustersize inference with random field and permutation methods. Neuroimage 2004;22: 676-687.

37. Whitfield-Gabrieli S, Nieto-Castanon A. Conn: a functional connectivity toolbox for correlated and anticorrelated brain networks. Brain Connect 2012;2:125-141.

38. Yeshokumar AK, Gordon-Lipkin E, Arenivas A, et al. Neurobehavioral outcomes in autoimmune encephalitis. J Neuroimmunol 2017;312:8-14.

39. Dubey D, Singh J, Britton JW, et al. Predictive models in the diagnosis and treatment of autoimmune epilepsy. Epilepsia 2017;58:1181-1189.

40. Parvizi J, Coburn KL, Shillcutt SD, Coffey CE, Lauterbach EC, Mendez MF. Neuroanatomy of pathological laughing and crying: a report of the American Neuropsychiatric Association Committee on Research. J Neuropsychiatry Clin Neurosci 2009;21:75-87.

41. Denckla CA, Fiori KL, Vingerhoets AJJM. Development of the crying proneness scale: associations among crying proneness, empathy, attachment, and age. J Pers Assess 2014;96:619-631.

42. Savage SA, Butler CR, Hodges JR, Zeman AZ. Transient epileptic amnesia over twenty years: long-term follow-up of a case series with three detailed reports. Seizure 2016;43:48-55.

43. Fanselow MS, Dong HWW. Are the dorsal and ventral hippocampus functionally distinct structures? Neuron 2010;65:7-19.

44. Immordino-Yang MH, McColl A, Damasio H, Damasio A. Neural correlates of admiration and compassion. Proc Natl Acad Sci USA 2009;106:8021-8026.

45. Beadle JN, Tranel D, Cohen NJ, Duff MC. Empathy in hippocampal amnesia. Front Psychol 2013;4:69.

46. McCormick C, Rosenthal CR, Miller TD, Maguire EA. Hippocampal damage increases deontological responses during moral decision making. J Neurosci 2016;36: 12157-12167.

47. Buckner RL, Krienen FM, Castellanos A, Diaz JC, Yeo BTT. The organization of the human cerebellum estimated by intrinsic functional connectivity. J Neurophysiol 2011;106:2322-2345.

48. Schmahmann JD, Pandya DN. Anatomical investigation of projections to the basis pontis from posterior parietal association cortices in rhesus monkey. J Comp Neurol 1989;289:53-73.

49. Platek SM, Wathne K, Tierney NG, Thomson JW. Neural correlates of self-face recognition: an effect-location meta-analysis. Brain Res 2008;1232:173-184.

50. Platek SM, Loughead JW, Gur RC, et al. Neural substrates for functionally discriminating self-face from personally familiar faces. Hum Brain Mapp 2006;27:91-98. 


\section{Neurology}

Pathologic tearfulness after limbic encephalitis: A novel disorder and its neural basis Georgios P.D. Argyropoulos, Lauren Moore, Clare Loane, et al.

Neurology 2020;94;e1320-e1335 Published Online before print January 24, 2020

DOI 10.1212/WNL.0000000000008934

This information is current as of January 24, 2020

\section{Updated Information \&} Services

References

Subspecialty Collections

\section{Permissions \& Licensing}

Reprints including high resolution figures, can be found at: http://n.neurology.org/content/94/12/e1320.full

This article cites 50 articles, 14 of which you can access for free at: http://n.neurology.org/content/94/12/e1320.full\#ref-list-1

This article, along with others on similar topics, appears in the following collection(s):

All Psychiatric disorders

http://n.neurology.org/cgi/collection/all_psychiatric_disorders Encephalitis

http://n.neurology.org/cgi/collection/encephalitis

\section{fMRI}

http://n.neurology.org/cgi/collection/fmri

Hippocampal sclerosis

http://n.neurology.org/cgi/collection/hippocampal_sclerosis

MRI

http://n.neurology.org/cgi/collection/mri

Information about reproducing this article in parts (figures,tables) or in its entirety can be found online at:

http://www.neurology.org/about/about_the_journal\#permissions

Information about ordering reprints can be found online:

http://n.neurology.org/subscribers/advertise

Neurology ${ }^{\circledR}$ is the official journal of the American Academy of Neurology. Published continuously since 1951, it is now a weekly with 48 issues per year. Copyright Copyright ( 2020 The Author(s). Published by Wolters Kluwer Health, Inc. on behalf of the American Academy of Neurology.. All rights reserved. Print ISSN: 0028-3878. Online ISSN: 1526-632X.

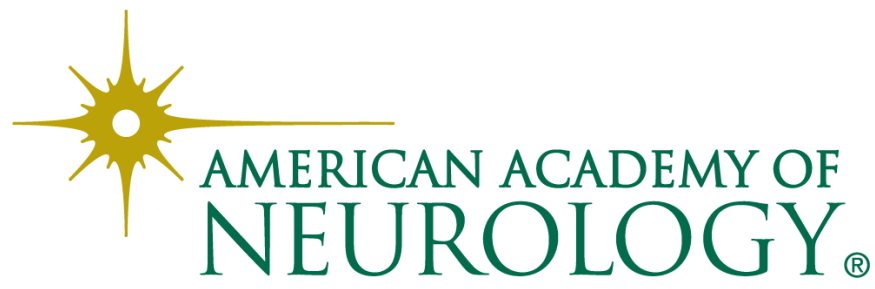

